\title{
Existence and multiplicity of positive solutions for a nonlocal differential equation
}

\author{
Yunhai Wang ${ }^{1 *}$, Fanglei Wang ${ }^{2,3}$ and Yukun $\mathrm{An}^{3}$
}

* Correspondence: yantaicity@163. com

${ }^{1}$ College of Aeronautics and Astronautics, Nanjing University of Aeronautics and Astronautics, Nanjing 210016, People's Republic of China

Full list of author information is available at the end of the article

\section{Abstract}

In this paper, the existence and multiplicity results of positive solutions for a nonlocal differential equation are mainly considered.

Keywords: Nonlocal boundary value problems, Cone, Fixed point theorem

\section{Introduction}

In this paper, we are concerned with the existence and multiplicity of positive solutions for the following nonlinear differential equation with nonlocal boundary value condition

$$
\left\{\begin{array}{l}
-\Phi\left(\int_{0}^{1}|u(s)|^{q} \mathrm{~d} \varphi(s)\right) u^{\prime \prime}(t)=h(t) f(u(t)), \quad \text { in } 0<t<1, \\
\alpha u(0)-\beta u^{\prime}(0)=0, \gamma u(1)+\delta u^{\prime}(1)=g\left(\int_{0}^{1} u(s) \mathrm{d} \varphi(s)\right),
\end{array}\right.
$$

where $\alpha, \beta, \gamma, \delta$ are nonnegative constants, $\rho=\alpha \gamma+\alpha \delta+\beta \gamma>0, q \geq 1$; $\int_{0}^{1}|u(s)|^{q} \mathrm{~d} \varphi(s), \int_{0}^{1}|u(s)|^{q} \mathrm{~d} \varphi(s)$ denote the Riemann-Stieltjes integrals.

Many authors consider the problem

$$
-\Delta u=M \frac{f(u)^{\alpha}}{\left(\int_{\Omega} f(u)\right)^{\beta}}, \text { in } \Omega \subset R^{n}, u=0, \quad \text { on } \partial \Omega \text {, }
$$

because of the importance in numerous physical models: system of particles in thermodynamical equilibrium interacting via gravitational potential, 2-D fully turbulent behavior of a real flow, one-dimensional fluid flows with rate of strain proportional to a power of stress multiplied by a function of temperature, etc. In [1,2], the authors use the Kras-noselskii fixed point theorem to obtain one positive solution for the following nonlocal equation with zero Dirichlet boundary condition

$$
-a\left(\int_{0}^{1}|u(s)|^{q}\right) u^{\prime \prime}(t)=h(t) f(u(t))
$$

when the nonlinearity $f$ is a sublinear or superlinear function in a sense to be established when necessary. Nonlocal BVPs of ordinary differential equations or system arise in a variety of areas of applied mathematics and physics. In recent years, more and more papers

(c) 2011 Wang et al; licensee Springer. This is an Open Access article distributed under the terms of the Creative Commons Attribution License (http://creativecommons.org/licenses/by/2.0), which permits unrestricted use, distribution, and reproduction in any medium, provided the original work is properly cited. 
were devoted to deal with the existence of positive solutions of nonlocal BVPs (see [3-9] and references therein). Inspired by the above references, our aim in the present paper is to investigate the existence and multiplicity of positive solutions to Equation 1 using the Krasnosel'skii fixed point theorem and Leggett-Williams fixed point theorem.

This paper is organized as follows: In Section 2, some preliminaries are given; In Section 3 , we give the existence results.

\section{Preliminaries}

Lemma 2.1 [3]. Let $y(t) \in C([0,1])$, then the problem

$$
\left\{\begin{array}{l}
-u^{\prime \prime}(t)=\gamma(t), \text { in } 0<t<1, \\
\alpha u(0)-\beta u^{\prime}(0)=0, \gamma u(1)+\delta u^{\prime}(1)=g\left(\int_{0}^{1} u(s) \mathrm{d} \varphi(s)\right),
\end{array}\right.
$$

has a unique solution

$$
u(t)=\frac{\beta+\alpha t}{\rho} g\left(\int_{0}^{1} u(s) \mathrm{d} \varphi(s)\right)+\int_{0}^{1} G(t, s) y(s) \mathrm{d} s,
$$

where the Green function $G(t, s)$ is

$$
G(t, s)=\frac{1}{\rho}, \begin{cases}(\beta+\alpha s)(\delta+\gamma-\gamma t), & \text { in } 0 \leq s \leq t \leq 1 \\ (\beta+\alpha t)(\delta+\gamma-\gamma s), & \text { in } 0 \leq t \leq s \leq 1\end{cases}
$$

It is easy to see that

$$
G(t, s)>0,0<t, s<1 ; G(t, s) \leq G(s, s), \quad 0 \leq t, s \leq 1,
$$

and there exists a $\theta \in\left(0, \frac{1}{2}\right)$ such that $G(t, s) \geq \theta G(s, s), \theta \leq t \leq 1-\theta, 0 \leq s \leq 1$.

For convenience, we assume the following conditions hold throughout this paper:

(H1) $f, g, \Phi: R^{+} \rightarrow R^{+}$are continuous and nondecreasing functions, and $\Phi(0)>0$;

$(\mathrm{H} 2) \phi(t)$ is an increasing nonconstant function defined on $[0,1]$ with $\phi(0)=0$;

(H3) $h(t)$ does not vanish identically on any subinterval of $(0,1)$ and satisfies

$$
0<\int_{\theta}^{1-\theta} G(t, s) h(s) \mathrm{d} s<+\infty .
$$

Obviously, $u \in C^{2}(0,1)$ is a solution of Equation 1 if and only if $u \in C(0,1)$ satisfies the following nonlinear integral equation

$$
u(t)=\frac{\beta+\alpha t}{\rho} g\left(\int_{0}^{1} u(s) \mathrm{d} \varphi(s)\right)+\int_{0}^{1} G(t, s) \frac{h(s) f(u(s))}{\Phi\left(\int_{0}^{1}|u|^{q} \mathrm{~d} \varphi\right)} d s .
$$

At the end of this section, we state the fixed point theorems, which will be used in Section 3.

Let $E$ be a real Banach space with norm $\|\cdot\|$ and $P \subset E$ be a cone in $E, P_{r}=\{x \in P$ : $\|x\|<r\}(r>0)$. Then, $\overline{P_{r}}=\{x \in P:\|x\| \leq r\}$. A map $\alpha$ is said to be a nonnegative continuous concave functional on $P$ if $\alpha: P \rightarrow[0,+\infty)$ is continuous and

$$
\alpha(t x+(1-t) y) \geq t \alpha(x)+(1-t) \alpha(y)
$$


for all $x, y \in P$ and $t \in[0,1]$. For numbers $a, b$ such that $0<a<b$ and $\alpha$ is a nonnegative continuous concave functional on $P$, we define the convex set

$$
P(\alpha, a, b)=\{x \in P: a \leq \alpha(x),\|x\| \leq b\} .
$$

Lemma 2.2 [10]. Let $A: \overline{P_{c}} \rightarrow \overline{P_{c}}$ be completely continuous and $\alpha$ be a nonnegative continuous concave functional on $P$ such that $\alpha(x)=\|x\|$ for all $x \in \overline{P_{c}}$. Suppose there exists $0<d<a<b=c$ such that

(i) $\{x \in P(\alpha, a, b): \alpha(x)>a\} \neq \varnothing$ and $\alpha(A x)>a$ for $x \in P(\alpha, a, b)$;

(ii) $\|A x\|<d$ for $\|x\| \leq d$;

(iii) $\alpha(A x)>a$ for $x \in P(\alpha, a, c)$ with $\|A x\|>b$.

Then, $A$ has at least three fixed points $x_{1}, x_{2}, x_{3}$ satisfying

$$
\begin{gathered}
\left\|x_{1}\right\|<d, \quad a<\alpha\left(x_{2}\right), \\
\left\|x_{3}\right\|>d \quad \text { and } \quad \alpha\left(x_{3}\right)<a .
\end{gathered}
$$

Lemma 2.3 [10]. Let $E$ be a Banach space, and let $P \subset E$ be a closed, convex cone in $E$, assume $\Omega_{1}, \Omega_{2}$ are bounded open subsets of $E$ with $0 \in \Omega_{1}, \bar{\Omega}_{1} \subset \Omega_{2}$, and $A: P \cap\left(\bar{\Omega}_{2} \backslash \Omega_{1}\right) \rightarrow P$ be a completely continuous operator such that either

(i) $\|A u\| \leq\|u\|, u \in P \cap \partial \Omega_{1}$ and $\|A u\| \geq\|u\|, u \in P \cap \partial \Omega_{2}$; or

(ii) $\|A u\| \geq\|u\|, u \in P \cap \partial \Omega_{1}$ and $\|A u\| \leq\|u\|, u \in P \cap \partial \Omega_{2}$.

Then, $A$ has a fixed point in $P \cap\left(\bar{\Omega}_{2} \backslash \Omega_{1}\right)$.

\section{Main result}

Let $E=C[0,1]$ endowed norm $\|u\|=\max _{0 \leq t \leq 1}|u|$, and define the cone $P \subseteq E$ by

$$
P=\left\{u \in E: u(t) \geq 0, \min _{\theta \leq t \leq 1-\theta} u(t) \geq \theta\|u\|\right\} .
$$

Then, it is easy to prove that $E$ is a Banach space and $P$ is a cone in $E$.

Define the operator $T: E \rightarrow E$ by

$$
T(u)(t)=\frac{\beta+\alpha t}{\rho} g\left(\int_{0}^{1} u(s) \mathrm{d} \varphi(s)\right)+\int_{0}^{1} G(t, s) \frac{h(s) f(u(s))}{\Phi\left(\int_{0}^{1}|u|^{q} \mathrm{~d} \varphi\right)} \mathrm{d} s .
$$

Lemma 3.1. $T: E \rightarrow E$ is completely continuous, and Te now prove that $P \subseteq P$.

Proof. For any $u \in P$, then from properties of $G(t, s), T(u)(t) \geq 0, t \in[0,1]$, and it follows from the definition of $T$ that

$$
\|T(u)\| \leq \frac{\alpha+\beta}{\rho} g\left(\int_{0}^{1} u(s) \mathrm{d} \varphi(s)\right)+\int_{0}^{1} G(s, s) \frac{h(s) f(u(s))}{\Phi\left(\int_{0}^{1}|u|^{q} \mathrm{~d} \varphi\right)} \mathrm{d} s .
$$

Thus, it follows from above that

$$
\begin{aligned}
\min _{\theta \leq t \leq 1-\theta} T(u)(t) & =\min _{\theta \leq t \leq 1-\theta}\left[\frac{\beta+\alpha t}{\rho} g\left(\int_{0}^{1} u(s) \mathrm{d} \varphi(s)\right)+\int_{0}^{1} G(t, s) \frac{h(s) f(u(s))}{\Phi\left(\int_{0}^{1}|u|^{q} \mathrm{~d} \varphi\right)} \mathrm{d} s\right] \\
& \geq \theta \frac{\alpha+\beta}{\rho} g\left(\int_{0}^{1} u(s) \mathrm{d} \varphi(s)\right)+\theta \int_{0}^{1} G(s, s) \frac{h(s) f(u(s))}{\Phi\left(\int_{0}^{1}|u|^{q} \mathrm{~d} \varphi\right)} \mathrm{d} s \\
& \geq \theta\|T(u)\|
\end{aligned}
$$


From the above, we conclude that $T P \subseteq P$. Also, one can verify that $T$ is completely continuous by the Arzela-Ascoli theorem.

Let

$$
\begin{gathered}
l=\min _{0 \leq t \leq 1} \int_{\theta}^{1-\theta} G(t, s) h(s) \mathrm{d} s, \quad L=\min _{\theta \leq t \leq 1-\theta} \int_{\theta}^{1-\theta} G(t, s) h(s) \mathrm{d} s, \\
\mathrm{~L}=\min _{0 \leq t \leq 1} \int_{0}^{1} G(t, s) h(s) \mathrm{d} s .
\end{gathered}
$$

Then, it is clear to see that $0<l \leq L<\mathrm{L}$.

Theorem 3.2. Assume (H1) to (H3) hold. In addition, (H4)

$$
\lim _{r \rightarrow 0^{+}} \inf \frac{f(\theta r)}{r \Phi\left(r^{q} \varphi(1)\right)} \geq \frac{1}{l} ;
$$

(H5) There exists a constant $2 \leq p_{1}$ such that

$$
\lim _{r \rightarrow \infty} \sup \frac{f(r)}{r \Phi\left((\varphi(1-\theta)-\varphi(\theta)) \theta^{q} r^{q}\right)} \leq \frac{1}{p_{1} \complement} ;
$$

(H6) There exists a constant $p_{2}$ with $\frac{1}{p_{1}}+\frac{1}{p_{2}}=1$ such that

$$
\lim _{r \rightarrow \infty} \sup \frac{g(r)}{r} \leq \frac{\rho}{p_{2} \varphi(1)(\beta+\alpha)}
$$

Then, problem (Equation 1) has one positive solution.

Proof. From (H4), there exists a $0<\eta<\infty$ such that

$$
\frac{f(\theta r)}{r \Phi\left(r^{q} \varphi(1)\right)} \geq \frac{1}{l}, \quad \forall 0<r \leq \eta .
$$

Choosing $R_{1} \in(0, \eta)$, set $\Omega_{1}=\left\{u \in E:\|u\|<R_{1}\right\}$. We now prove that

$$
\|T u\| \geq\|u\|, \quad \forall u \in P \cap \partial \Omega_{1} .
$$

Let $u \in P \cap \partial \Omega_{1}$. Since $\min _{\theta \leq t \leq 1-\theta} u(t) \geq \theta\|u\|$ and $\|u\|=R_{1}$, from Equation 3, (H1) and (H3), it follows that

$$
\begin{aligned}
T u(t) & =\frac{\beta+\alpha t}{\rho} g\left(\int_{0}^{1} u(s) \mathrm{d} \varphi(s)\right)+\int_{0}^{1} G(t, s) \frac{h(s) f(u(s))}{\Phi\left(\int_{0}^{1}|u|^{q} \mathrm{~d} \varphi\right)} \mathrm{d} s \\
& \geq \int_{0}^{1} G(t, s) \frac{h(s) f(u(s))}{\Phi\left(\int_{0}^{1}|u|^{q} \mathrm{~d} \varphi\right)} \mathrm{d} s \\
& \geq \int_{\theta}^{1-\theta} G(t, s) \frac{h(s) f(u(s))}{\Phi\left(\int_{0}^{1}|u|^{q} \mathrm{~d} \varphi\right)} \mathrm{d} s \\
& \geq \frac{f\left(\theta R_{1}\right)}{\Phi\left(R_{1}^{q} \varphi(1)\right)} \int_{\theta}^{1-\theta} G(t, s) h(s) \mathrm{d} s \\
& \geq \frac{f\left(\theta R_{1}\right)}{\Phi\left(R_{1}^{q} \varphi(1)\right)} l \\
& \geq R_{1}=\|u\| .
\end{aligned}
$$


Then, Equation 4 holds.

On the other hand, from (H5), there exists $\overline{R_{1}}>0$ such that

$$
\frac{f(r)}{r \Phi\left((\varphi(1-\theta)-\varphi(\theta)) \theta^{q} r^{q}\right)} \leq \frac{1}{p_{1} \iota^{\prime}}, \quad \forall r \geq \overline{R_{1}} .
$$

From (H6), there exists $\overline{R_{2}}>0$ such that

$$
\frac{g(r)}{r} \leq \frac{\rho}{p_{2} \varphi(1)(\beta+\alpha)}, \quad \forall r \geq \overline{R_{2}} .
$$

Choosing $R_{2}=\max \left\{R_{1}, \overline{R_{1}}, \frac{\overline{R_{2}}}{\theta(\varphi(1-\theta)-\varphi(\theta))}\right\}+1$, set $\Omega_{2}=\left\{u \in E:\|u\|<R_{2}\right\}$. We now prove that

$$
\|T u\| \leq\|u\|, \quad \forall u \in P \cap \partial \Omega_{2} .
$$

If $u \in P \cap \partial \Omega_{2}$, we have

$$
\int_{1}^{0} u(s) \mathrm{d} \varphi(s) \geq \int_{\theta}^{1-\theta} u(s) \mathrm{d} \varphi(s) \geq \theta R_{2}(\varphi(1-\theta)-\varphi(\theta)) \geq \overline{R_{2}} .
$$

From Equations 5, 6, we can prove

$$
\begin{aligned}
T u(t) & =\frac{\beta+\alpha t}{\rho} g\left(\int_{0}^{1} u(s) \mathrm{d} \varphi(s)\right)+\int_{0}^{1} G(t, s) \frac{h(s) f(u(s))}{\Phi\left(\int_{0}^{1}|u|^{q} \mathrm{~d} \varphi\right)} \mathrm{d} s \\
& \leq \frac{\beta+\alpha}{\rho} g\left(\int_{0}^{1} u(s) \mathrm{d} \varphi(s)\right)+\int_{0}^{1} G(t, s) \frac{h(s) f(u(s))}{\Phi\left(\int_{0}^{1}|u|^{q} \mathrm{~d} \varphi\right)} \mathrm{d} s \\
& \leq \frac{\beta+\alpha}{\rho} \frac{\rho}{p_{2} \varphi(1)(\beta+\alpha)} \int_{0}^{1} u(s) \mathrm{d} \varphi(s)+f(\|u\|) \int_{0}^{1} G(t, s) \frac{h(s)}{\Phi\left(\int_{\theta}^{1-\theta}|u|^{q} \mathrm{~d} \varphi\right)} \mathrm{d} s \\
& \leq \frac{\beta+\alpha}{\rho} \frac{\rho}{p_{2} \varphi(1)(\beta+\alpha)}\|u\| \varphi(1)+\frac{f(\|u\|)}{\Phi\left((\varphi(1-\theta)-\varphi(\theta)) \theta^{q}\|u\|^{q}\right)} \int_{0}^{1} G(t, s) h(s) \mathrm{d} s \\
& \leq \frac{R_{2}}{p_{1}}+\frac{R_{2}}{p_{2}} \\
& =R_{2}=\|u\| .
\end{aligned}
$$

Then, Equation 7 holds.

Therefore, by Equations 4 and 7 and the second part of Lemma 2.3, $T$ has a fixed point in $P \cap\left(\bar{\Omega}_{2} \backslash \Omega_{1}\right)$, which is a positive solution of Equation 1 .

Example. Let $q=2, h(t)=1, \Phi(s)=2+s, \phi(t)=2 t, f(u)=\frac{\theta^{2}(1-2 \theta)}{4 \mathrm{~L}}\left(u^{\frac{1}{3}}+u^{3}\right)$ and $g(s)=s^{\frac{1}{2}}$, namely,

$$
\left\{\begin{array}{l}
-\left(2+\int_{0}^{1}|u(s)|^{2} \mathrm{~d}(2 s)\right) u^{\prime \prime}(t)=\frac{\theta^{2}(1-2 \theta)}{4 \mathrm{~L}}\left(u^{\frac{1}{3}}+u^{3}\right), \quad \text { in } 0<t<1, \\
\alpha u(0)-\beta u^{\prime}(0)=0, \gamma u(1)+\delta u^{\prime}(1)=\left[\int_{0}^{1} u(s) \mathrm{d}(2 s)\right]^{\frac{1}{2}} .
\end{array}\right.
$$


It is easy to see that (H1) to (H3) hold. We also can have

$$
\begin{gathered}
\lim _{r \rightarrow 0+} \inf \frac{f(\theta r)}{r \Phi\left(r^{q} \varphi(1)\right)}=\lim _{r \rightarrow 0+} \inf \frac{\frac{\theta^{2}(1-2 \theta)}{4 \ell}\left((\theta r)^{\frac{1}{3}}+(\theta r)^{3}\right)}{r\left(2+2 r^{2}\right)}=\infty, \\
\lim _{r \rightarrow \infty} \sup \frac{f(r)}{r \Phi\left((\varphi(1-\theta)-\varphi(\theta)) \theta^{q} r^{q}\right)}=\lim _{r \rightarrow \infty} \sup \frac{\frac{\theta^{2}(1-2 \theta)}{4 \ell}\left(r^{\frac{1}{3}}+r^{3}\right)}{r\left(2+2(1-2 \theta) \theta^{2} r^{2}\right)}=\frac{1}{8 \ell} .
\end{gathered}
$$

Take $p_{1}=2$, then it is clear to see that (H4) and (H5) hold. Since

$$
\lim _{r \rightarrow \infty} \sup \frac{g(r)}{r}=\lim _{r \rightarrow \infty} \sup \frac{r^{\frac{1}{2}}}{r}=0,
$$

then (H6) hold.

Theorem 3.3. Assume (H1) to (H3) hold. In addition,

(H7) There exists a constant $2 \leq p_{1}$ such that

$$
\lim _{r \rightarrow 0} \sup \frac{f(r)}{r \Phi\left((\varphi(1-\theta)-\varphi(\theta)) \theta^{q} r^{q}\right)} \leq \frac{1}{p_{1} \longleftarrow} ;
$$

(H8) There exists a constant $p_{2}$ with $\frac{1}{p_{1}}+\frac{1}{p_{2}}=1$ such that

$$
\lim _{r \rightarrow 0} \sup \frac{g(r)}{r} \leq \frac{\rho}{p_{2} \varphi(1)(\beta+\alpha)} ;
$$

(H9)

$$
\lim _{r \rightarrow \infty} \inf \frac{f(\theta r)}{r \Phi\left(r^{q} \varphi(1)\right)} \geq \frac{1}{l} .
$$

Then, problem (Equation 1) has one positive solution.

Proof. From (H7), there exists $\eta_{1}>0$ such that

$$
\frac{f(r)}{r \Phi\left((\varphi(1-\theta)-\varphi(\theta)) \theta^{q} r^{q}\right)} \leq \frac{1}{p_{1} \ell^{\prime}}, \quad \forall 0<r<\eta_{1} .
$$

From (H8), there exists $\eta_{2}>0$ such that

$$
\frac{g(r)}{r} \leq \frac{\rho}{p_{2} \varphi(1)(\beta+\alpha)}, \quad \forall 0<r<\eta_{2} .
$$

Choosing $R_{1}=\min \left\{\eta_{1}, \frac{\eta_{2}}{\varphi(1)}\right\}$, set $\Omega_{1}=\left\{u \in E:\|u\|<R_{1}\right\}$. We now prove that

$$
\|T u\| \leq\|u\|, \quad \forall u \in P \cap \partial \Omega_{1} .
$$

If $u \in P \cap \partial \Omega_{1}$, we have

$$
\int_{0}^{1} u(s) \mathrm{d} \varphi(s) \leq \int_{0}^{1} R_{1} \mathrm{~d} \varphi(s) \leq R_{1} \varphi(1) \leq \eta_{2} .
$$


From Equations 8, 9, we can prove

$$
\begin{aligned}
T u(t) & =\frac{\beta+\alpha t}{\rho} g\left(\int_{0}^{1} u(s) \mathrm{d} \varphi(s)\right)+\int_{0}^{1} G(t, s) \frac{h(s) f(u(s))}{\Phi\left(\int_{0}^{1}|u|^{q} \mathrm{~d} \varphi\right)} \mathrm{d} s \\
& \leq \frac{\beta+\alpha}{\rho} g\left(\int_{0}^{1} u(s) \mathrm{d} \varphi(s)\right)+\int_{0}^{1} G(t, s) \frac{h(s) f(u(s))}{\Phi\left(\int_{0}^{1}|u|^{q} \mathrm{~d} \varphi\right)} \mathrm{d} s \\
& \leq \frac{\beta+\alpha}{\rho} \frac{\rho}{p_{2} \varphi(1)(\beta+\alpha)} \int_{0}^{1} u(s) \mathrm{d} \varphi(s)+f(\|u\|) \int_{0}^{1} G(t, s) \frac{h(s)}{\Phi\left(\int_{\theta}^{1-\theta}|u|^{q} \mathrm{~d} \varphi\right)} \mathrm{d} s \\
& \leq \frac{\beta+\alpha}{\rho} \frac{\rho}{p_{2} \varphi(1)(\beta+\alpha)}\|u\| \varphi(1)+\frac{f(\|u\|)}{\Phi\left((\varphi(1-\theta)-\varphi(\theta)) \theta^{q}\|u\|^{q}\right)} \int_{0}^{1} G(t, s) h(s) \mathrm{d} s \\
& \leq \frac{R_{1}}{p_{1}}+\frac{R_{1}}{p_{2}} \\
& =R_{1}=\|u\| .
\end{aligned}
$$

Then, Equation 10 holds.

On the other hand, from (H7), there exists $\overline{R_{1}}>0$ such that

$$
\frac{f(\theta r)}{r \Phi\left(r^{q} \varphi(1)\right)} \geq \frac{1}{l}, \quad \forall r \geq \overline{R_{1}} .
$$

Choosing $R_{2}=\max \left\{R_{1},\left(\frac{\overline{R_{1}}}{\theta^{q}(\varphi(1-\theta)-\varphi(\theta))}\right)^{\frac{1}{q}}\right\}+1$, set $\Omega_{2}=\left\{u \in E:\|u\|<R_{2}\right\}$. We now prove that

$$
\|T u\| \geq\|u\|, \quad \forall u \in P \cap \partial \Omega_{2} .
$$

If $u \in P \cap \partial \Omega_{2}$, Since $\min _{\theta \leq t \leq 1-\theta} u(t) \geq \theta\|u\|$ and $\|u\|=R_{2}$, we have

$$
\int_{0}^{1}|u|^{q} \mathrm{~d} \varphi(s) \geq \int_{\theta}^{1-\theta}|u|^{q} \mathrm{~d} \varphi \geq \theta^{q} R_{2}^{q}(\varphi(1-\theta)-\varphi(\theta)) \geq \overline{R_{1}} .
$$

By Equation 11, (H1) and (H3), it follows that

$$
\begin{aligned}
T u(t) & =\frac{\beta+\alpha t}{\rho} g\left(\int_{0}^{1} u(s) \mathrm{d} \varphi(s)\right)+\int_{0}^{1} G(t, s) \frac{h(s) f(u(s))}{\Phi\left(\int_{0}^{1}|u|^{q} \mathrm{~d} \varphi\right)} \mathrm{d} s \\
& \geq \int_{0}^{1} G(t, s) \frac{h(s) f(u(s))}{\Phi\left(\int_{0}^{1}|u|^{q} \mathrm{~d} \varphi\right)} \mathrm{d} s \\
& \geq \int_{\theta}^{1-\theta} G(t, s) \frac{h(s) f(u(s))}{\Phi\left(\int_{0}^{1}|u|^{q} \mathrm{~d} \varphi\right)} \mathrm{d} s \\
& \geq \frac{f\left(\theta R_{2}\right)}{\Phi\left(R_{2}^{q} \varphi(1)\right)} \int_{\theta}^{1-\theta} G(t, s) h(s) \mathrm{d} s \\
& \geq \frac{f\left(\theta R_{2}\right)}{\Phi\left(R_{2}^{q} \varphi(1)\right)} l \\
& \geq R_{2}=\|u\| .
\end{aligned}
$$


Then, Equation 12 holds.

Therefore, by Equations 10 and 12 and the first part of Lemma 2.3, $T$ has a fixed point in $P \cap\left(\bar{\Omega}_{2} \backslash \Omega_{1}\right)$, which is a positive solution of Equation 1.

Example. Let $q=2, h(t)=t, \Phi(s)=2+s, \phi(t)=2 t, f(u)=\frac{2}{l \theta^{3}} u^{2}$ and $g(s)=s^{2}$.

Theorem 3.4. Assume that (H1) to (H3) hold. In addition, $\phi(1) \geq 1$, and the functions $f, g$ satisfy the following growth conditions:

(H10)

$$
\begin{gathered}
\lim _{r \rightarrow \infty} \sup \frac{f(r)}{\Phi\left((\varphi(1-\theta)-\varphi(\theta)) \theta^{q} r^{q}\right) r}<\frac{1}{4 \mathrm{~L}}, \\
\lim _{r \rightarrow \infty} \sup \frac{g(r)}{r}<\frac{\rho}{4(\beta+\alpha) \varphi(1)}
\end{gathered}
$$

(H11)

$$
\begin{gathered}
\lim _{r \rightarrow 0} \sup \frac{f(r)}{\Phi\left((\varphi(1-\theta)-\varphi(\theta)) \theta^{q} r^{q}\right) r}<\frac{1}{2 \ell} \\
\lim _{r \rightarrow 0} \sup \frac{g(r)}{r}<\frac{\rho}{2(\beta+\alpha) \varphi(1)}
\end{gathered}
$$

(H12) There exists a constant $a>0$ such that

$$
f(u)>\frac{\Phi\left(\left(\frac{a}{\theta}\right)^{q} \varphi(1)\right) a}{L}, \quad \text { for } u \in\left[a, \frac{a}{\theta}\right]
$$

Then, BVP (Equation 1) has at least three positive solutions.

Proof. For the sake of applying the Leggett-Williams fixed point theorem, define a functional $\sigma(u)$ on cone $P$ by

$$
\sigma(u)=\min _{\theta \leq t \leq 1-\theta} u(t), \quad \forall u \in P .
$$

Evidently, $\sigma: P \rightarrow R^{+}$is a nonnegative continuous and concave. Moreover, $\sigma(u) \leq \|$ $u \|$ for each $u \in P$.

Now, we verify that the assumption of Lemma 2.2 is satisfied.

Firstly, it can verify that there exists a positive number $c$ with $c \geq b=\frac{a}{\theta}$ such that $T: \overline{P_{c}} \rightarrow P_{c}$.

By (H10), it is easy to see that there exists $\tau>0$ such that

$$
\begin{gathered}
\frac{f(r)}{\Phi\left((\varphi(1-\theta)-\varphi(\theta)) \theta^{q} r^{q}\right) r}<\frac{1}{4 \mathrm{~L}}, \quad \forall r \geq \tau, \\
\frac{g(r)}{r}<\frac{\rho}{4(\beta+\alpha) \varphi(1)}, \quad \forall r \geq \tau,
\end{gathered}
$$

Set

$$
M_{1}=\frac{f(\tau)}{\Phi(0)}, \quad M_{2}=g(\tau) .
$$

Taking

$$
c>\max \left\{b, 4 \ell M_{1}, \frac{4 M_{2}(\beta+\alpha)}{\rho}\right\} .
$$


If $u \in \overline{P_{c}}$, then

$$
\begin{aligned}
\|T u(t)\| & =\max _{t \in[0,1]}|T u(t)| \\
& =\max _{t \in[0,1]} \frac{\beta+\alpha t}{\rho} g\left(\int_{0}^{1} u(s) \mathrm{d} \varphi(s)\right)+\max _{t \in[0,1]} \int_{0}^{1} G(t, s) \frac{h(s) f(u(s))}{\Phi\left(\int_{0}^{1}|u|^{q} \mathrm{~d} \varphi\right)} \mathrm{d} s \\
& \leq \frac{\beta+\alpha}{\rho} g\left(\int_{0}^{1} u(s) \mathrm{d} \varphi(s)\right)+\max _{t \in[0,1]}^{1} G(t, s) \frac{h(s) f(u(s))}{\Phi\left(\int_{0}^{1}|u|^{q} \mathrm{~d} \varphi\right)} \mathrm{d} s \\
& \leq \frac{\beta+\alpha}{\rho} g(\varphi(1)\|u\|)+\max _{t \in[0,1]} \frac{f(\|u\|)}{\Phi\left((\varphi(1-\theta)-\varphi(\theta)) \theta^{q}\|u\|^{q}\right)} \int_{0}^{1} G(t, s) h(s) \mathrm{d} s \\
& \leq \frac{\beta+\alpha}{\rho}\left(\frac{\rho}{4(\beta+\alpha) \varphi(1)} \varphi(1)\|u\|+M_{2}\right)+\left\llcorner\left(\frac{\|u\|}{4 \mathrm{~L}}+M_{1}\right)\right. \\
& <c .
\end{aligned}
$$

by (H1) to (H3) and (H10).

Next, from (H11), there exists $d^{\prime} \in(0, a)$ such that

$$
\begin{gathered}
\frac{f(r)}{\Phi\left((\varphi(1-\theta)-\varphi(\theta)) \theta^{q} r^{q}\right) r}<\frac{1}{2 \ell}, \quad \forall r \in\left[0, d^{\prime}\right], \\
\frac{g(r)}{r}<\frac{\rho}{2(\beta+\alpha) \varphi(1)}, \quad \forall r \in\left[0, d^{\prime}\right] .
\end{gathered}
$$

Take $d=\frac{d^{\prime}}{\varphi(1)}$. Then, for each $u \in \overline{P_{d}}$, we have

$$
\begin{aligned}
\|T u(t)\| & =\max _{t \in[0,1]}|T u(t)| \\
& =\max _{t \in[0,1]} \frac{\beta+\alpha t}{\rho} g\left(\int_{0}^{1} u(s) \mathrm{d} \varphi(s)\right)+\max _{t \in[0,1]} \int_{0}^{1} G(t, s) \frac{h(s) f(u(s))}{\Phi\left(\int_{0}^{1}|u|^{q} \mathrm{~d} \varphi\right)} \mathrm{d} s \\
& \leq \frac{\beta+\alpha}{\rho} g\left(\int_{0}^{1} u(s) \mathrm{d} \varphi(s)\right)+\max _{t \in[0,1]} \int_{0}^{1} G(t, s) \frac{h(s) f(u(s))}{\Phi\left(\int_{0}^{1}|u|^{q} \mathrm{~d} \varphi\right)} \mathrm{d} s \\
& \leq \frac{\beta+\alpha}{\rho} g(\varphi(1)\|u\|)+\max _{t \in[0,1]} \frac{f(\|u\|)}{\Phi\left((\varphi(1-\theta)-\varphi(\theta)) \theta^{q}\|u\|^{q}\right)} \int_{0}^{1} G(t, s) h(s) \mathrm{d} s \\
& \leq \frac{\beta+\alpha}{\rho}\left(\frac{\rho}{2(\beta+\alpha) \varphi(1)} \varphi(1)\|u\|\right)+\leftarrow\left(\frac{\|u\|}{2 \ell}\right) \\
& <d .
\end{aligned}
$$

Finally, we will show that $\{u \in P(\sigma, a, b): \sigma(u)>a\} \neq \varnothing$ and $\sigma(T u)>a$ for all $u \in P$ $(\sigma, a, b)$.

In fact,

$$
u(t)=\frac{a+b}{2} \in\{u \in P(\sigma, a, b): \sigma(u)>a\} .
$$

For $u \in P(\sigma, a, b)$, we have

$$
b \geq\|u\| \geq u \geq \min _{t \in[\theta, 1-\theta]} u(t) \geq a,
$$


for all $t \in[\theta, 1-\theta]$. Then, we have

$$
\begin{aligned}
\min _{t \in[\theta, 1-\theta]} T u(t) & =\min _{t \in[\theta, 1-\theta]} \frac{\beta+\alpha t}{\rho} g\left(\int_{0}^{1} u(s) \mathrm{d} \varphi(s)\right)+\min _{t \in[\theta, 1-\theta]} \int_{0}^{1} G(t, s) \frac{h(s) f(u(s))}{\Phi\left(\int_{0}^{1}|u|^{q} \mathrm{~d} \varphi\right)} \mathrm{d} s \\
& \geq \min _{t \in[\theta, 1-\theta]} \int_{0}^{1} G(t, s) \frac{h(s) f(u(s))}{\Phi\left(\int_{0}^{1}|u|^{q} \mathrm{~d} \varphi\right)} \mathrm{d} s \\
& \geq \frac{1}{\Phi\left(\varphi(1) b^{q}\right)} \min _{t \in[\theta, 1-\theta]} \int_{\theta}^{1-\theta} G(t, s) h(s) f(u(s)) \mathrm{d} s \\
& \left.>\frac{1}{\Phi\left(\varphi(1) b^{q}\right)} \frac{\Phi\left(b^{q} \varphi(1)\right) a}{L} \min _{t \in[\theta, 1-\theta]} \int_{\theta}^{1-\theta} G(t, s) h(s)\right) \mathrm{d} s \\
& =a
\end{aligned}
$$

by (H1) to (H3), (H12). In addition, for each $u \in P(\theta, a, c)$ with $\|T u\|>b$, we have

$$
\min _{t \in[\theta, 1-\theta]}(T u)(t) \geq \theta\|T u\|>\theta b \geq a .
$$

Above all, we know that the conditions of Lemma 2.2 are satisfied. By Lemma 2.2, the operator $T$ has at least three fixed points $u_{i}(i=1,2,3)$ such that

$$
\begin{gathered}
\left\|u_{1}\right\|<d, \\
a<\min _{t \in[\theta, 1-\theta]} u_{2}(t) \\
\left\|u_{3}\right\|>d \text { with } \min _{t \in[\theta, 1-\theta]} u_{3}(t)<a .
\end{gathered}
$$

The proof is complete.

Example. Let $q=2, h(t)=t, \Phi(s)=2+s, \phi(t)=2 t, f(u)=4 \frac{1+\theta^{2}}{L \theta^{2}} u^{2}$ and, $g(s)=\frac{\rho}{16(\beta+\alpha)} \frac{s^{2}}{2+s}$, namely,

$$
\left\{\begin{array}{l}
-\left(2+\int_{0}^{1}|u(s)|^{2} \mathrm{~d}(2 s)\right) u^{\prime \prime}(t)=t 4 \frac{1+\theta^{2}}{l \theta^{2}} u^{2}, \text { in } 0<t<1, \\
\alpha u(0)-\beta u^{\prime}(0)=0, \gamma u(1)+\delta u^{\prime}(1)=\frac{\rho}{16(\beta+\alpha)} \frac{\left(\int_{0}^{1} u(s) \mathrm{d}(2 s)\right)^{2}}{2+\int_{0}^{1} u(s) \mathrm{d}(2 s)} .
\end{array}\right.
$$

From a simple computation, we have

$$
\begin{gathered}
\lim _{r \rightarrow \infty} \sup \frac{f(r)}{\Phi\left((\varphi(1-\theta)-\varphi(\theta)) \theta^{2} r^{2}\right) r}=\lim _{r \rightarrow \infty} \sup \frac{4 \frac{1+\theta^{2}}{L \theta^{2}} r^{2}}{\left(2+2(1-2 \theta) \theta^{2} r^{2}\right) r}=0, \\
\lim _{r \rightarrow \infty} \sup \frac{g(r)}{r}=\lim _{r \rightarrow \infty} \sup \frac{\frac{\rho}{16(\beta+\alpha)} \frac{r^{2}}{2+r}}{r}=\frac{\rho}{16(\beta+\alpha)}<\frac{\rho}{4(\beta+\alpha) \varphi(1)}, \\
\limsup _{r \rightarrow 0} \frac{f(r)}{\Phi\left((\varphi(1-\theta)-\varphi(\theta)) \theta^{q} r^{q}\right) r}=\lim _{r \rightarrow 0} \sup \frac{4 \frac{1+\theta^{2}}{L \theta^{2}} r^{2}}{\left(2+2(1-2 \theta) \theta^{2} r^{2}\right) r}=0, \\
\limsup _{r \rightarrow 0} \frac{g(r)}{r}=\lim _{r \rightarrow 0} \sup \frac{\frac{\rho}{16(\beta+\alpha)} \frac{r^{2}}{2+r}}{r}=0,
\end{gathered}
$$

Then, it is easy to see that (H1) to (H3) and (H10) to (H11) hold. Especially, take $a=$ 1, by $f(a)=f(1)=4 \frac{1+\theta^{2}}{L \theta^{2}}>2 \frac{1+\theta^{2}}{L \theta^{2}}=\frac{\Phi\left(\left(\frac{a}{\theta}\right)^{a} \varphi(1)\right) a}{L}$ and (H1), then (H12) holds. 


\section{Author details}

'College of Aeronautics and Astronautics, Nanjing University of Aeronautics and Astronautics, Nanjing 210016, People's Republic of China ${ }^{2}$ College of Science, Hohai University, Nanjing 210098, People's Republic of China ${ }^{3}$ Department of Mathematics, Nanjing University of Aeronautics and Astronautics, Nanjing 210016, People's Republic of China

\section{Authors' contributions}

In this manuscript the authors studied the existence and multiplicity of positive solutions for an interesting nonlocal differential equation using the Cone-Compression and Cone-Expansion Theorem due to M. Krasnosel'skii for the existence result and Leggett-Williams fixed point Theorem for the multiplicity result. Moreover, in this work, the authors supplements the studies done in [12], because here they consider the case nonlocal boundary value condition. All authors typed, read and approved the final manuscript.

\section{Competing interests}

The authors declare that they have no competing interests.

Received: 21 February 2011 Accepted: 11 July 2011 Published: 11 July 2011

\section{References}

1. Correa, FJSA: On positive solutions of nonlocal and nonvariational elliptic problems. Nonliear Anal. 59, 1147-1155 (2004)

2. Stanczy, R: Nonlocal elliptic equations. Nonlinear Anal. 47, 3579-3548 (2001). doi:10.1016/S0362-546X(01)00478-3

3. Kang, P, Wei, Z: Three positive solutions of singular nonlocal boundary value problems for systems of nonlinear second-order ordinary differential equations. Nonlinear Anal. 70, 444-451 (2009). doi:10.1016/j.na.2007.12.014

4. Kang, P, Xub, J, Wei, Z: Positive solutions for $2 \mathrm{p}$-order and $2 q$-order systems of singular boundary value problems with integral boundary conditions. Nonlinear Anal. 72, 2767-2786 (2010). doi:10.1016/j.na.2009.11.022

5. Perera, K, Zhang, Z: Nontrivial solutions of Kirchhoff type problems via the Yang index. J Diff Equ. 221 (1), 246-255 (2006). doi:10.1016/j.jde.2005.03.006

6. Pietramala, P: A note on a beam equation with nonlinear boundary conditions. Boundary Value Problems 2011, 14 (2011). (Article ID 376782)

7. Wang, F, An, Y: Existence of nontrivial solution for a nonlocal elliptic equation with nonlinear boundary condition Boundary Value Problems 2009, 8 (2009). (Article ID 540360)

8. Webb, JRL, Infante, G: Non-local boundary value problems of arbitrary order. J Lond Math Soc. 79, 238-258 (2009)

9. Zhang, Z, Perera, K: Sign-changing solutions of Kirchhoff type problems via invariant sets of descent flow. J Math Anal Appl. 317 (2), 456-463 (2006). doi:10.1016/j.jmaa.2005.06.102

10. Guo, D, Lakshmikantham, V: Nonlinear Problems in Abstract Cones. Academic Press, Orlando (1988)

doi:10.1186/1687-2770-2011-5

Cite this article as: Wang et al.: Existence and multiplicity of positive solutions for a nonlocal differential equation. Boundary Value Problems 2011 2011:5.

\section{Submit your manuscript to a SpringerOpen ${ }^{\odot}$ journal and benefit from:}

- Convenient online submission

- Rigorous peer review

- Immediate publication on acceptance

- Open access: articles freely available online

- High visibility within the field

- Retaining the copyright to your article

Submit your next manuscript at $\gg$ springeropen.com 